\title{
Caracterización y valoración económica en sistemas agroforesales con cacao (Theobroma cacao L.) en los departamentos de Amazonas y San Martin
}

\section{Characterization and economic valuation in agroforestry systems with cocoa (Theobroma cacao L.) in the departments of Amazonas and San Martin}

\author{
Sandra Zumaeta Villanueva ${ }^{1}$, Anthony Spencer Díaz Chira ${ }^{2}$
}

\section{RESUMEN}

Los departamentos de Amazonas y San Martin presentan un alto índice de diversidad de especies el cual, juega un rol importante en la producción de bienes y servicios ecosistémicos, asociados al desarrollo de productos emblemáticos como el cacao. El estudio realizó la caracterización y valorización económica en sistemas agroforestales (SAF) con cacao, obteniendo dos grupos, con guaba" y sin guaba; con guaba estando asociado con frutales y sin guaba encontrándose asociada con especies maderables siendo estos clasificados a su vez en corto, mediano y largo plazo; se valoraron económicamente los SAF encontrados y relacionados a la biodiversidad, obteniendo como resultado para el departamento de San Martin, los siguientes ingresos netos promedio según caracterización: con guaba obteniendo un ingreso neto de $\mathrm{S} / 49$ $760.5889 \mathrm{Ha} / \mathrm{año}$ y un ingreso neto promedio para asociaciones sin guaba de S/ $72447.6667 \mathrm{Ha} /$ año y en el departamento de Amazonas encontramos un ingreso neto promedio con guaba de S/ $48443.3 \mathrm{Ha} /$ año y sin guaba de S/ 72 173.65. Ha/año.

Palabras clave: Sistema agroforestal, valoración económica, cacao.

\begin{abstract}
The departments of Amazonas and San Martin present a high index of species diversity which plays an important role in the production of ecosystem goods and services, associated with the development of emblematic products such as cocoa. The study carried out the characterization and economic valuation in agroforestry systems (SAF) with cocoa, obtaining two groups, with guaba and without it; with guaba being associated with fruit trees and without guaba being associated with timber species being these classified in turn in short, medium and long term; the SAF found and related to biodiversity were valued economically, obtaining as a result for the department of San Martin, the following average net income according to characterization: with guava obtaining a net income of S / $49760.5889 \mathrm{Ha} /$ year and an average net income for associations without guava of S / $72447.6667 \mathrm{Ha} /$ year and in the department of Amazonas we found an average net income with guava of S / $48443.3 \mathrm{Ha} /$ year and without guaba of S / $72173.65 \mathrm{Ha}$ /year
\end{abstract}

Key words: Agroforestry system, economic valuation, cocoa

\footnotetext{
${ }^{1}$ Bachiller en Ingeniería Agrónoma sazuvi93@hotmail.com

2 Bachiller en Ingeniería Ambiental diazchira24@hotmail.com
} 


\section{INTRODUCCIÓN}

El cultivo de cacao en los departamentos de Amazonas y San Martin, es de mucha importancia para la economía familiar del ámbito rural, pues los pequeños agricultores, además del café y de otros frutales, lo incluyen como parte integrante de la unidad productiva diversificada.

En la actualidad en el departamento de Amazonas se estima que existan alrededor de $13483 \mathrm{Ha} \mathrm{de}$ cacao, obteniendo rendimientos que oscilan de $550 \mathrm{Kg}$ a $650 \mathrm{Kg} / \mathrm{Ha}$. Esta actividad involucra a 11644 agricultores cacaoteras aproximadamente (IV Censo Nacional Agropecuario, 2012). Los distritos de mayor producción son Copallin, la Peca e Imaza en la provincia de Bagua.

Así mismo en el departamento de San Martin se ha estimado que existan alrededor de $46915 \mathrm{Ha}$ instaladas a nivel de toda la región, con un rendimiento promedio de $730 \mathrm{Kg}$ de cacao seco/Ha y una producción registrada de 5991.34 TM (IV Censo Nacional Agropecuario, 2012), involucrando a 25927 agricultores, siendo la zona de mayor producción las provincias de Lamas, Mariscal Cáceres, Moyobamba, Bellavista, El Dorado, etc.

El cacao es una de las materias primas agrícolas más importantes del comercio internacional; como tal es una fuente indispensable de divisas para muchos países. Se estima la producción mundial en más de siete millones de hectáreas y unas 3.7 millones de toneladas según la Organización Internacional del Cacao (ICCO). Entre los mayores productores se encuentran Costa de Marfil (33\%), Indonesia (18\%) y Ghana $(15 \%)$. En Centro América la producción de cacao representa menos del $1 \%$ de la producción mundial, pero es de gran importancia para las familias rurales cacaoteras indígenas y mestizas por ser uno de sus principales ingresos económicos y su relevancia sociocultural (Estrada, 2010).

Desde el punto de vista agroecológico, el cacao tiene un alto valor como prestador de servicios ecosistémicos que aún no son reconocidos; las plantaciones fomentan la biodiversidad y contribuyen a la conservación de las cuencas; alimentan la vida de ríos y arroyos, proporcionan agua para consumo humano y animal, para el riego de cultivos, y para generación de energía hidroeléctrica. Además, juega un rol importante en la mitigación de los efectos del calentamiento y el cambio climático: una plantación almacena en la biomasa aérea entre 60 y $10 \mathrm{Tn}$ de carbono/Ha. (López et al., 2015).

Según J, Sánchez y A, Dubón, 2003; el programa de Cacao y Agroforestería, que comprende un área de $200 \mathrm{Ha}$, promueve otros sistemas rentables y protectores de los recursos naturales, como el asocio de especies maderables: laurel y cedro, o frutales perennes como el rambután. En los últimos 12 años el asocio con cedro mantiene el mejor promedio de producción $(10389 \mathrm{Kg} / \mathrm{Ha})$. El asocio con rambután presenta una producción acumulada de $9700 \mathrm{Kg} / \mathrm{Ha}$, el asocio con laurel presenta una producción acumulada de 8014 $\mathrm{Kg} / \mathrm{Ha}$; y la asociación con leguminosas que antiguamente realizaba el agricultor presenta una producción de $9084 \mathrm{Kg} / \mathrm{Ha}$. La producción de rambután en el año 2002 fue de 2550 frutos/árbol, para una producción proyectada por hectárea de 204000 frutos, en una población productiva de 80 árboles/Ha. El laurel y el cedro después de quince años de establecidos estos asocios, alcanzaron un diámetro promedio de 46.4 y $41.7 \mathrm{~cm}$, respectivamente. La producción anual de madera por hectárea es de $133 \mathrm{~m}^{3} / \mathrm{ha}$ en el laurel y 90 $\mathrm{m}^{3} / \mathrm{Ha}$. en el cedro. En el supuesto de que al 
decimoquinto año fuera aprovechado la madera, más los ingresos acumulados por cacao, el productor estaría obteniendo ingresos brutos aproximados de Lps. 400000 y Lps. 430 000/Ha en los asocios con laurel, cedro y rambután, respectivamente, mientras que en el sistema tradicional (cacao con sombra de leguminosas), le generaría solamente un ingreso bruto promedio anual en el mismo periodo de Lps. 10 770/Ha.

Los sistemas forestales son diversos y con altos niveles de biodiversidad estos proveen diversos servicios ecológicos, aumentan los procesos ecológicos tales como el control de plagas y la polinización, a menudo con altos rendimientos económicos, no solo son una forma ecológica sostenible de producir cultivos, sino que también proveen fuentes de entradas y recursos adicionales a los agricultores como son la producción de leña, frutas y pasto a la vez protegen la biodiversidad de los efectos negativos generados por el uso y abuso de químicos en la agricultura $\mathrm{y}$ monocultivos.

La agroforestería se ha planteado como uno de los sistemas de uso de la tierra, en el cual las especies arbóreas, en combinación espacial o secuencial en el tiempo, crecen junto con cultivos agrícolas tal es el caso del cacao, proporcionando mayores beneficios para el suelo, con el mantenimiento y mejoramiento de la fertilidad por medio del reciclaje de nutrientes, reducción del riesgo de erosión eólica e hídrica.

Con el manejo de los sistemas agroforestales se verá beneficiado el sector productivo de los departamentos de Amazonas y San Martin específicamente en los distritos de Imaza y Lamas respectivamente.

\section{MATERIAL Y MÉTODOS}

\section{a. Diseño de investigación}

El diseño de la investigación fué no experimental, particularmente es un diseño transversal, cuyo diseño transversal es puramente descriptivo comparativo, ya que tuvo como objetivo indagar la incidencia y los valores en que se manifiestan una o más variables.

\section{b. Población}

La población estuvo conformada por el total de productores cacaoteros (unidades agropecuarias - U.A) del distrito de Lamas y de Imaza

Tabla 1. Población de productores cacaoteros

\begin{tabular}{|l|l|l|}
\hline DEPARTAMENTO & LUGAR & U.A \\
\hline San Martin & Lamas & 2625 \\
\hline Amazonas & Imaza & 6505 \\
\hline
\end{tabular}

\section{c. Muestra, muestreo}

Para la selección de la muestra en los productores cacaoteros de las provincias de Lamas e Imaza, se utilizó el muestreo de tipo probabilístico, empleando la siguiente fórmula.

$$
n_{0}=\frac{\sum_{h=1}^{L} W_{h} p_{h}\left(1-p_{h}\right)}{\frac{e^{2}}{Z_{(1-\alpha / 2)}^{2}}+\frac{1}{N} \sum_{h=1}^{L} W_{h} p_{h}\left(1-p_{h}\right)}
$$

Donde:

$\mathrm{p}_{\mathrm{h}}$ : Proporción de UA que en el estrato $\mathrm{h}$ 1- $\alpha$ : Nivel de confianza.

$\mathrm{Z}_{(1-\alpha / 2)}$ : Cuantil de la distribución Normal Estándar.

e: Margen de error.

Obteniendo como muestra de la investigación: 
Tabla 2. Muestra de productores cacaoteros

\begin{tabular}{|c|c|c|c|}
\hline DEPART. & PROVIN. & CULTIVO & $\mathbf{n}_{\mathbf{i}}$ \\
\hline San Martin & Lamas & Cacao & 31 \\
\hline Amazonas & Bagua & Cacao & 77 \\
\hline \multicolumn{3}{|c|}{ TOTAL } & 108 \\
\hline
\end{tabular}

d. Métodos, técnicas e instrumentos de recolección de datos

La investigación se desarrolló en tres fases que se detallan a continuación:

\section{Fase de recopilación}

La recopilación de la información preliminar se realizó mediante entrevistas con los responsables de las instituciones involucradas, así como también con personas naturales que vienen implementando los sistemas agroforestales.

\section{Fase de campo}

- Diagnóstico del lugar y aplicación de pre encuesta

Durante esta etapa se realizó las coordinaciones correspondientes acerca de los productores cacaoteros según la muestra de estudio y se aplicó una pre encuesta para determinar el lugar de estudio.

- Visitas, evaluación de experiencias locales y observación de los sistemas agroforestales.

Las visitas se realizaron a las parcelas de los productores cacaoteros con sistemas agroforestales y con sistema tradicional, para observar los diferentes tipos de arreglos en los sistemas.

- Aplicación de encuestas a los agricultores cacaoteros

La encuesta aplicada a los agricultores consistió una secuencia de preguntas relacionadas al terreno en general., a la parcela de cacao con o sin sistemas agroforestales y a las características básicas de la casa y hogar del productor.

\section{Fase de gabinete}

Se realizó mediante la caracterización de los sistemas agroforestales propuesta según Ospina, 2004. El cual presenta tres categorías: sistemas agroforestales, técnicas agroforestales y prácticas agroforestales, para la valoración económica se utilizó el método de cambio de productividad (valor de uso directo) empleando las siguientes formulas:

Costo por actividad $=\#$ jornales $\mathrm{x}$ precio de jornal (costo de mano de obra de actividades)

Costos de insumos en actividades $=$ precio de insumos $\mathrm{x}$ costo de insumos

Costo producción = suma de $\operatorname{costos}$ por actividad + costo de insumos en actividad Ingreso $=$ precio cacao $\mathrm{x}$ producción anual Ingreso neto-beneficio $=$ ingreso - costo de producción.

\section{RESULTADOS}

3.1. Caracterización de los sistemas agroforestales

a. Tipificación de los sistemas
agroforestales

Se identificaron 11 especies maderables; descritas en la tabla 3, en el departamento de Amazonas y San Martin, donde se realizó una clasificación de dos grupos Con Guaba y Sin Guaba; considerando a los Con Guaba como asociación de cacao con frutales y Sin Guaba a la asociación de cacao con árboles maderables de corto, mediano y largo plazo. 
Tabla 3. Lista de especies agroforestales asociados al cultivo de cacao en los departamentos de Amazonas y San Martin

\begin{tabular}{|c|c|c|c|c|}
\hline \multicolumn{3}{|c|}{ CLASIFICACIóN } & \multirow{2}{*}{$\frac{\text { FAMILIA }}{\text { MUSACEAE }}$} & \multirow{2}{*}{$\begin{array}{l}\text { NOMBRE COMUN } \\
\text { Musa paradisiaca }\end{array}$} \\
\hline \multirow{4}{*}{$\begin{array}{l}\text { Con } \\
\text { guaba }\end{array}$} & \multirow{4}{*}{ FrutalesS } & PLATANO & & \\
\hline & & MANGO & ANACARDIACEAE & Mangifera indica \\
\hline & & NARANJA & RUTACEAE & Citrus sinensis \\
\hline & & AGUAJE & ARACACEAE & Mauritia flexus \\
\hline \multirow{11}{*}{$\begin{array}{l}\text { Sin } \\
\text { Guaba }\end{array}$} & \multirow{11}{*}{ Maderables } & BOLAINA & MALVACEAE & Guazuma crinita \\
\hline & & CAPIRONA & RUBIACEAE & $\begin{array}{l}\text { Calycophyllum } \\
\text { multiflorum }\end{array}$ \\
\hline & & RUFINDI & FABACEAE & Inga ruiziana \\
\hline & & ISHPINGO & FABACEAE & Amburana cearesis \\
\hline & & SHAINA & RHAMNACEAE & Colubrina gladulosa \\
\hline & & TORNILLO & MIMOSACEAE & $\begin{array}{l}\text { Cedrelinga } \\
\text { catenaeformis }\end{array}$ \\
\hline & & $\begin{array}{l}\text { PALO } \\
\text { BLANCO }\end{array}$ & RUBIACEAE & $\begin{array}{l}\text { Calycophyllum } \\
\text { multiflorum }\end{array}$ \\
\hline & & CEDRO & MELIACEAE & Cedrela sp. \\
\hline & & PALIPERRO & VERBENACEAE & Vitex pseudolea \\
\hline & & CAOBA & MELIACEAE & $\begin{array}{l}\text { Swietenia } \\
\text { macrophylla }\end{array}$ \\
\hline & & $\begin{array}{l}\text { PINO } \\
\text { CHUNCHO }\end{array}$ & CAESALPINOIDEAE & $\begin{array}{l}\text { Schizolobium } \\
\text { amazonicum }\end{array}$ \\
\hline
\end{tabular}

Tabla 4. Tipificación de los sistemas agroforestales en Amazonas y San Martin

\begin{tabular}{|l|l|r|r|}
\hline \multicolumn{2}{|c|}{ TIPIFICACIÓN } & AMAZONAS & SAN MARTIN \\
\hline \multirow{3}{*}{ CON GUABA } & FRUTALES & 40 & 22 \\
\hline \multirow{3}{*}{ SIN GUABA } & CORTO & 16 & 16 \\
\cline { 2 - 4 } & MEDIANO & 14 & 9 \\
\cline { 2 - 4 } & LARGO & 9 & 11 \\
\hline
\end{tabular}

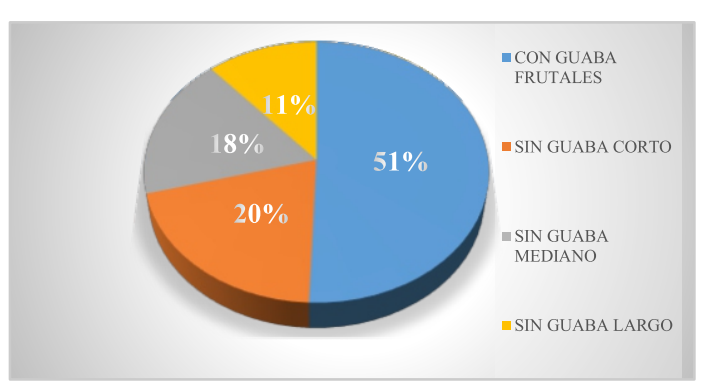

Figura 1. Tipificación de sistemas agroforestales Amazonas y San Martin

En la tipificación en los departamentos de Amazonas y San Martin nos muestra en la figura 1 que el $51 \%$ de la muestra total entre Amazonas y San Martin son productores que presentan una asociación del cultivo de cacao con guaba y frutales, así como también el 20\%, 18\% y 11\% de la muestra total de ambos departamentos tienen sistemas asociados el cultivo de cacao con árboles maderables de corto, media y largo plazo respectivamente, teniendo en cuenta que se puede encontrar de 1 a 11 especies maderables por cada parcela evaluada.

Así también en ambos departamentos se encontraron los siguientes arreglos: árboles en asociación con cultivos perennes/frutales, arreglos maderables plantados al cuadro y cercos vivos.

\section{b. Valoración económica en los departamentos de Amazonas y San Martin.}

Tabla 5. Ingresos netos de la caracterización de Amazonas y San Martin

\begin{tabular}{|c|c|c|c|}
\hline \multicolumn{4}{|c|}{ INGRESO NETO (S/ Ha/año) } \\
\hline $\begin{array}{c}\text { CARACTERI_ } \\
\text { ZACIóN }\end{array}$ & AÑO & AMAZONAS & SAN MARTN \\
\hline \multirow{3}{*}{ CON GUABA } & 2014 & - & 28532.8 \\
\cline { 2 - 4 } & 2015 & 35067.5 & 42718.6 \\
\cline { 2 - 4 } & 2016 & 61819.1 & 78030.37 \\
\hline \multirow{3}{*}{ SIN GUABA } & 2014 & - & 28481.25 \\
\cline { 2 - 4 } & 2015 & 57225.6 & 44395.25 \\
\cline { 2 - 4 } & 2016 & 87121.7 & 144466.5 \\
\hline
\end{tabular}

En la tabla 5 se detalla los ingresos netos de los productores de acuerdo a la producción obtenida en los tres últimos años, 2014, 2015 y 2016. Para el departamento de Amazonas no se consideró el año 2014 ya que el productor cacaotero manifestó que en ese año no obtuvo producción por presencia de enfermedades como por ejemplo la moniliasis (Moniliphthora roreri).

Para obtener un ingreso mejor detallado para una unidad agrícola se elaboró la tabla. 6 , donde nos indica los ingresos netos promedios, teniendo en cuenta una hectárea por familia, tanto por año como por mes. 
Tabla 6. Ingresos netos por familia de la caracterización en los departamentos de Amazonas y San Martin

\begin{tabular}{|c|c|c|}
\hline \multicolumn{3}{|c|}{ INGRESO NETO POR FAMILIA (S/Ha) } \\
\hline PERIODO & AMAZONAS & $\begin{array}{c}\text { SAN } \\
\text { MARTÍN }\end{array}$ \\
\hline AÑO & 1566.45 & 2842.05 \\
\hline MES & 130.54 & 236.84 \\
\hline
\end{tabular}

\section{c. Variables sensibles:}

Las variables sensibles encontradas que influyen directamente en el ingreso del productor cacaotero es el costo de la mano de obra y el precio del cacao $/ \mathrm{kg}$. Demostrándonos que si el costo de la mano de obra se incrementa esta afectara a los ingresos del productor, así como el precio del cacao disminuye se obtendrá perdidas económicas, como se observa en la tabla 7 y 8.

Tabla 7. Variable sensible mano de obra

\begin{tabular}{|c|c|}
\hline MANO DE OBRA & INGRESO NETO \\
\hline$\Delta 30 \%$ & 500 \\
\hline$\Delta 35 \%$ & 250 \\
\hline$\Delta 40 \%$ & 0 \\
\hline$\Delta 45 \%$ & -250 \\
\hline$\Delta 50 \%$ & -500 \\
\hline
\end{tabular}

Tabla 8. Variable sensible precio cacao/kg

\begin{tabular}{|c|c|}
\hline PRECIO S/ & INGRESO NETO \\
\hline$\downarrow 5.00$ & 325 \\
\hline$\downarrow 4.50$ & 175 \\
\hline$\downarrow 4.00$ & 25 \\
\hline$\downarrow 3.75$ & 0 \\
\hline$\downarrow 3.50$ & -125 \\
\hline$\downarrow 3.00$ & -275 \\
\hline
\end{tabular}

\section{DISCUSIÓN}

El estudio tuvo por fin relacionar el potencial agroforestal asociado al cultivo de cacao con el valor que representa para el productor. Por tal motivo se trata de demostrar la mejora de producción del cultivo de cacao asociado a un sistema agroforestal. Se discute la participación del productor en la actividad y la significancia de la disponibilidad inmediata del recurso agroforestal, los servicios ambientales $\mathrm{y}$ productivos del sistema para su retribución en forma económica.

- La Asociación de Sistemas Agroforestales con cacao tienen un gran desempeño con la rentabilidad de dicho cultivo; como el caso de Amazonas que se encontró que su ingreso promedio neto es de S/ $120616.95 \mathrm{Ha} /$ año, obteniendo a la vez un ingreso por familia de S/ 1566.45 por año en sistemas agroforestales con guaba, tal como lo indica MINCETUR (2008), el cultivo de cacao en el departamento de Amazonas es de mucha importancia para la economía familiar del ámbito rural, pues los pequeños agricultores, además del café y otros frutales, lo incluyen como parte integrante de la unidad productiva diversificada.

- En San Martin el ingreso promedio neto fue de S/ $122208.28 \mathrm{Ha} /$ año, teniendo un ingreso por familia de $\mathrm{S} / 2842.05$ por año en sistemas agroforestales con guaba, lo que no demuestra la investigación de W. Guzmán. 2015. Que el incremento de la rentabilidad con sistemas agroforestales (SA) incluyen una retribución de casi $\mathrm{S} / 1000$ por año a una tasa de $10 \%$.

- Los arreglos forestales encontrados tanto en el departamento de Amazonas y San Martin 
son: el cultivo de cacao asociado a cultivos perennes y árboles forestales como el cedro, ishpingo, caoba, capirona, entre otros; así mismo se presencio el arreglo tradicional del cultivo de cacao con guaba y frutales. Sobre la base de lo antes indicado y considero en campo. Según, W. Guzmán, 2015. Que encontró parecidos arreglos forestales que responden a sistemas agroforestales simultáneos del tipo silvoagricultura, ya que en general tanto para San Martin y Amazonas el cultivo de cacao aparece siempre acompañado de árboles perennes como, por ejemplo; Capirona, laurel, cedro o bolaina, existiendo también arreglos forestales con guaba y frutales tanto en Alto Mayo y Juanjuí.

- La participación de la mano de obra en el proceso del cultivo de cacao regula la organización de la finca. Mediante el presente estudio podemos destacar que el $98 \%$ de la responsabilidad productiva es familiar en el departamento de Amazonas, en cambio en San Martin el $60 \%$ de responsabilidad productiva es mano rentada, manifestando que al incremento de la mano de obra se produce una perdida económica, como lo corrobora la investigación de $W$. Guzmán, 2015. Teniendo los costos de mano de obra y la baja productividad en los departamentos de Amazonas y San Martin estarían conllevando a rentabilidades negativas.

\section{CONCLUSIONES}

El potencial de los sistemas agroforestales radica en la capacidad de integrar especies maderables como acompañamiento al cultivo principal, aun cuando los beneficios no sean directos, el sistema puede aportar numerables ventajas productivas al cultivo principal además de la función básica de cobertura vegetal. Así mismo brinda soporte energético a las familias y el aporte de poder diversificar la ración alimenticia con frutas de la finca.

De acuerdo al resultado se demuestra a ver encontrado 11 especies maderables de corto, mediano y largo plazo de crecimiento que presentan un valor forestal-maderable, mientras que también encontramos parcelas asociadas únicamente con guaba y frutales. Dentro de estas especies maderables encontramos algunas con valor comercial como el cedro y otras especies en menos cantidad que aportan efectos secundarios a la productividad del sistema según el objetivo del productor.

En el departamento de San Martin en los tres últimos años 2014, 2015 y 2016; se obtuvo un beneficio total con guaba de $\mathrm{S} / 28532.8 \mathrm{Ha} /$ año, S/ $42718.6 \mathrm{Ha} /$ año y S/ $78030.37 \mathrm{Ha} / \mathrm{año}$; respectivamente, y un ingreso neto total sin guaba S/ $28481.25 \mathrm{Ha} /$ año, S/ $44395.25 \mathrm{Ha} /$ año y S/ $144466.5 \mathrm{Ha} / a n ̃ o$, el cual nos demuestra que la asociación de sistemas agroforestales con cacao genera mejores ingresos al productor cacaotero, así mismo, en el departamento de Amazonas durante los dos últimos años, 2015 y 2016; se obtuvo un beneficio total con guaba S/ 92293.1

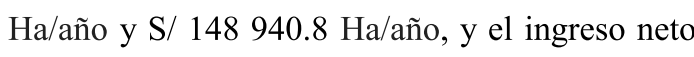
sin guaba es de S/ $57225.6 \mathrm{Ha} /$ año y S/ 87121.7 $\mathrm{Ha} / a n ̃ o$, demostrando igualmente que los sistemas agroforestales son rentables para el agricultor cacaotero.

Las variables más sensibles encontradas; en el costo de producción es la mano de obra sabiendo 
que si esté incrementa al $45 \%$ los ingresos disminuirán obteniendo perdidas económicas; en cambio para el ingreso la siguiente variable sensible encontrada es el Precio de Venta del producto, sabiendo que si este disminuye a un precio menor de $\mathrm{S} / 3.50 \mathrm{Kg} /$ cacao, el beneficio del productor se verá afectada. (Ver tablas 7 y 8).

\section{REFERENCIA BIBLIOGRAFICA}

- Estrada Garro, J. 2010. "Balance de Nutrientes en sistemas agroforestales de cacao (Theobroma cacao) orgánico". Nicaragua. Tesis. Mg. Agricultura Ecológica. Escuela de Posgrado. Centro Agronómico Tropical de Investigación y Enseñanza. Pág. 1.

- Guzmán, W. 2015. “Análisis costo beneficio económico, social y ambiental de sistemas de producción sostenibles de cacao en las regiones de Amazonas y San Martín”. Cooperación Alemana, implementada por la GIZ. ProAmbiente-Perú. Amazonas.

- López, O.; S. I, Ramírez.; S, Espinoza.; J, Villareal y A, Wong. 2015. "Diversidad vegetal y sustentabilidad del sistema agroforestal de cacao". Revista Iberoamericana de Ciencias. México. Vol. 2. $\mathrm{N}^{\circ}$ 2. marzo 2015. Pág. 1.

- MINCETUR, 2008. Estudio de caracterización del potencial genético de cacao en el Perú. Proyecto de Cooperación UE-Perú en materia de asistencia técnica relativa al comercio. $\mathrm{M}$ and $\mathrm{O}$ Consulting SAC, Empresa Consultora.

- Sánchez, J \& A. Dubón, 2003. "Programa de cacao y agroforestería” Fundación Hondureña de Investigación Agrícola. Honduras. Febrero 2003. Pág. 7.
- Ospina, A. 2004. "Propuesta Metodológica de Clasificación Agroforestal". Fundación Ecovivero. Colombia. 